\title{
RF NOISE DRIVEN DIPOLE MODE PARAMETRIC RESONANCE
}

\author{
Yujong Kim*, C. Kim, K. H. Kim, I. H. Yu, J. S. Yang, M. H. Chun \\ M. Kwon, J. Y. Huang, S. H. Nam, M. H. Cho, W. Namkung, and I. S. Ko \\ Pohang Accelerator Laboratory, POSTECH, Pohang, 790-784, Korea
}

\begin{abstract}
In the Pohang Light Source (PLS) storage ring, we have occasionally experienced the abnormal spontaneous beam lifetime reduction and strong longitudinal damping. Due to the abnormal beam lifetime reduction, available beam time is reduced substantially. From the recent data analysis of the PLS longitudinal feedback system (LFS) and the streak camera, we have found that the abnormal phenomena can be generated by the RF noise via the dipole mode parametric resonance of the RF phase modulation. We describe the observation of the dipole mode parametric resonance driven by the RF noise and compare it with a simulation result in the PLS storage ring.
\end{abstract}

\section{INTRODUCTION}

After changing the operation condition of the Pohang Light Source (PLS) storage ring from 400 bucket filled mode to full 468 bucket filled one at $2.5 \mathrm{GeV}$, we have occasionally met the abnormal large reduction in the beam lifetime and strong longitudinal stability. Also, the transverse beam instability would be generated at the much lower beam current due to the longitudinally stable beams. Although we did not change any machine parameter and always turned off the active longitudinal feedback system (LFS), these abnormal beam lifetime reduction, the strong longitudinal damping, and the lowered threshold of the transverse beam instability had been spontaneously happened during the user beam service period. The phenomena had been generated randomly about two times per a month. Although the magnitude of the beam lifetime reduction and the strength of the longitudinal damping were changed slowly, their abnormal properties were maintained for a few tens of hour once the phenomena were generated. Due to the abnormal phenomena, available beam time was reduced substantially, and we had injected beams more often than the normal operation. To analyze these phenomena and solve the problem, we had monitored the beam motion with the streak camera and the LFS by using it as a data acquisition system (DAQ) for the beam diagnostics [1]. From their various data, we have found that the abnormal phenomena have relation with the RF phase modulation due to the RF noise-sidebands in the No. 3 low level RF system. In this paper, we have described the RF noise driven dipole mode parametric resonance of the RF phase modulation in the PLS storage ring.

\footnotetext{
*yjkim@POSTECH.edu
}

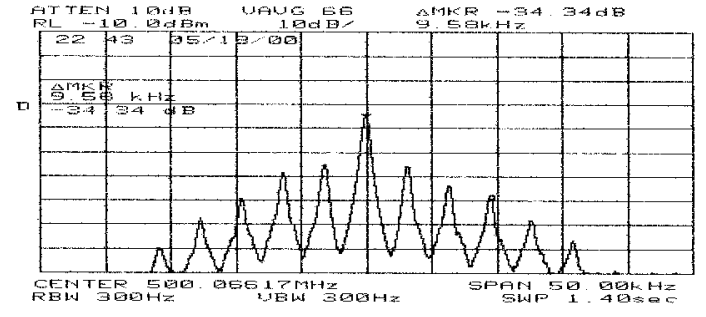

Figure 1: RF noise-sidebands in the No. 3 low level RF system when the abnormal beam lifetime reduction and the longitudinal damping are generated.

\section{RF PHASE MODULATION}

\subsection{Noise in Low Level RF System}

During the normal operation period, there is no RF noise-sideband around the RF frequency of $500.066 \mathrm{MHz}$. However, when the abnormal beam lifetime reduction and longitudinal damping are happened, there are always the RF noise-sidebands in the No. 3 low level RF system as shown in Fig. 1. Note that the third RF noise-sideband of Fig. 1 is close to the synchrotron frequency of $9.773 \mathrm{kHz}$ at $2.5 \mathrm{GeV}$, and the same sideband pattern with different level is also observed in the beam position monitor (BPM) signal via the LFS DAQ and the spectrum analyzer. Therefore, these noise-sidebands work as the source of the abnormal beam lifetime reduction and longitudinal damping. Since the sideband pattern around the RF frequency is similar to that of the RF phase modulation, we can consider the noise-sidebands as the modulation signals of the RF phase modulation [2].

\subsection{Dipole Mode Parametric Resonance}

When the modulation frequency of the RF phase modulation is in resonance with an odd multiple of the synchrotron frequency, the oscillating components due to the RF phase modulation are out of phase with the synchrotron oscillations. We call this resonance as the parametric resonance of the RF phase modulation. More details about theory and simulation of the parametric resonance can be found in the reference [3] and references therein. The dipole mode parametric resonance can be generated when one of the RF noise-sidebands with enough amplitude is near the synchrotron frequency [2], [3]. In this case, various beam properties can be changed nonlinearly according to the relation of the RF phase modulation frequency $f_{m}$ 


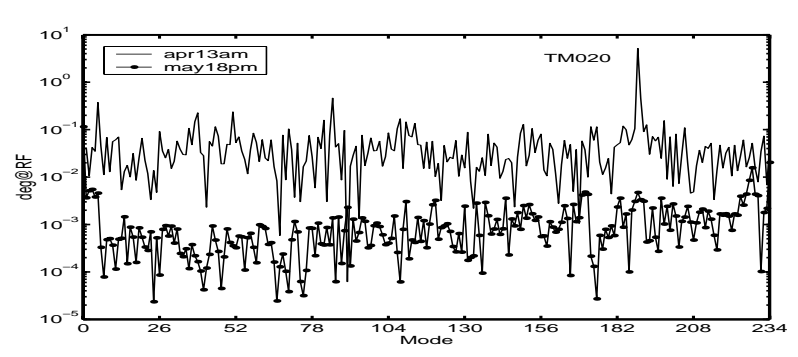

(a)

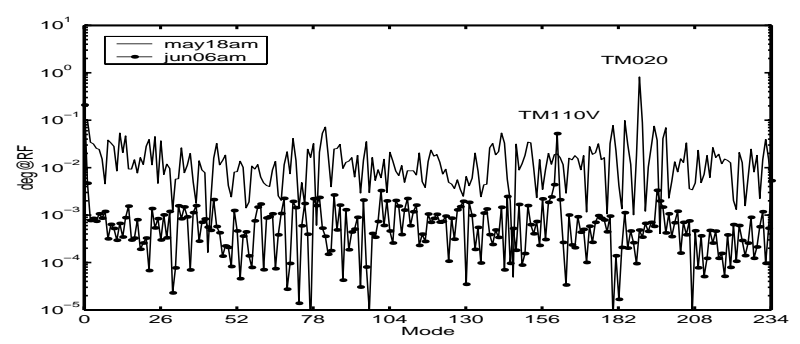

(b)

Figure 2: Spectrum levels of the CBMI's obtained by the LFS DAQ for four different RF noise-sideband conditions; (a) apr13am and may18pm, (b) mayl8am and jun06am.

and the bifurcation frequency $f_{c}$ defined as

$$
f_{c}=f_{s}\left(1-\frac{3}{16}\left(4 a_{m}\right)^{2 / 3}\right) \text {. }
$$

Here, $f_{s}$ is the synchrotron frequency, and $a_{m}$ is the amplitude of the RF phase modulation [3]. Although the beams can be modulated by all RF noise-sidebands in Fig. 1, all bifurcation frequencies of five RF noise-sidebands in Fig. 1 are about $9 \mathrm{kHz}$ for the given modulation amplitude distribution. Therefore, the beam stability and the bunch length can be nonlinearly controlled by only a noise-sideband close to $9 \mathrm{kHz}$ via the dipole mode parametric resonance of the RF phase modulation. From now on, the modulation frequency $f_{m}$ means the frequency of an RF noisesideband close to $9 \mathrm{kHz}$.

\subsection{Beam Instability and Beam Lifetime}

Although the longitudinal coupled bunch mode instabilities (LCBMI's) due to the higher order modes (HOM's) of the PLS RF cavities can be controlled by the fine RF cavity temperature tuning system, there are still an undamped harmful LCBMI due to $\mathrm{TM}_{020} \mathrm{HOM}(1301.1 \mathrm{MHz})$ of the PLS RF cavities during all 468 bucket filled $2.5 \mathrm{GeV}$ normal operation period as shown in aprl3am of Fig. 2(a) [1]. Here, aprl3am denotes the measured time, that is April 13 AM, 2000, and "deg@RF" means the bunch phase [degree] with respect to a reference oscillator of the PLS LFS [1]. Due to the large momentum spread, the beam lifetime of aprl3am is larger than 36 hours at $160.00 \mathrm{~mA}$ as shown in Fig. 3 [2], [4]. However, the beam lifetime, LCBMI, and the transverse coupled bunch mode instability (TCBMI) of

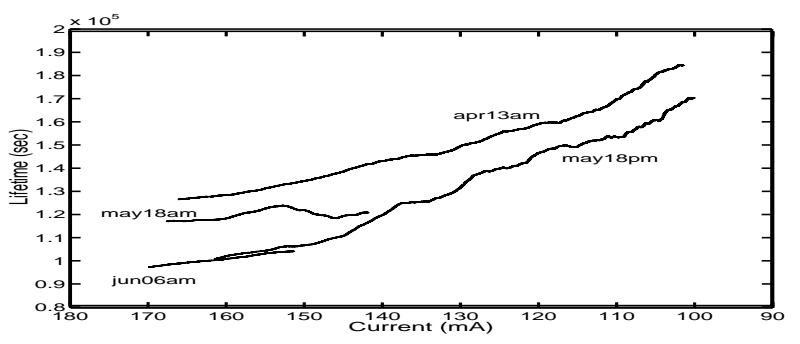

Figure 3: Beam lifetime for the normal operation period, aprl3am and abnormal beam lifetime reduction due to the various RF noise-sidebands

Table 1: Parameters for various RF noise status.

\begin{tabular}{lcccc}
\hline & apr13am & may18am & may18pm & jun06am \\
\hline Beam energy, GeV & 2.5 & 2.5 & 2.5 & 2.5 \\
Beam current, mA & 159.18 & 159.26 & 159.13 & 165.80 \\
$f_{s}, \mathrm{kHz}$ & 9.773 & 9.773 & 9.773 & 9.773 \\
$f_{m}, \mathrm{kHz}$ & no noise & 8.369 & 9.662 & 10.380 \\
$a_{m}, \mathrm{rad}$ &. & 0.006 & 0.003 & 0.002 \\
$f_{c}, \mathrm{kHz}$ &. & 9.615 & 9.672 & 9.700 \\
$f_{m}-f_{c}, \mathrm{kHz}$ &. & -1.246 & -0.010 & +0.680 \\
Peak mode of LCBMI & 279 & 279 & 241 & 195 \\
Peak mode of TCBMI &. &. &. & 161 \\
LCBMI level, deg@RF & 5.500 & 0.850 & 0.015 & 0.003 \\
TCBMI level, deg@RF &. &. &. & 0.06 \\
Beam lifetime, hour:min & $36 \mathrm{~h} 36 \mathrm{~m}$ & $32 \mathrm{~h} 57 \mathrm{~m}$ & $27 \mathrm{~h} 40 \mathrm{~m}$ & $26 \mathrm{~h} 47 \mathrm{~m}$ \\
\hline
\end{tabular}

may18am, may18pm, and jun06am are changed according to different modulation frequencies as shown in Figs. 2 and 3. Related parameters are summarized in Table 1.

To analyze those changes for the mayl8am, mayl8pm, and jun06am, we have simulated the dipole mode parametric resonance by solving the time averaged Hamilton equations of motion in the resonant rotating frame with the modulation frequency [2], [3]. Figure 4 shows the 100000 turn phase space tracking results in the resonant rotating frame for may18am and may18pm. Here, all notations are the same as those of the reference [3], and we have simulated under the same initial conditions. As the modulation frequency approaches the bifurcation frequency from below, one stable fixed point $\mathrm{A}$ and one unstable fixed point $\mathrm{C}$ move in and the other stable fixed point $\mathrm{B}$ moves out, and the momentum spread or the bunch length will also be reduced as shown in Fig. 4 [2]-[4]. Finally, the stable fixed point $\mathrm{B}$ and the unstable fixed point $\mathrm{C}$ become one at the bifurcation frequency [2], [3]. Since two beamlets around two stable fixed points oscillate out of phase in the lab coordinate frame, and enough electrons can be diffused from one beamlet to the other beamlet at $f_{c}$, the net dipole mode phase oscillations in the bunches will be disappeared at the dipole mode parametric resonance condition of $f_{m}=f_{c}$ [2], [3]. In this case, the driving mechanism of the LCBMI's will be vanished, and the strong longitudinal stability and the reduction in the momentum spread or the bunch length reduction will be obtained [2]-[4]. There- 


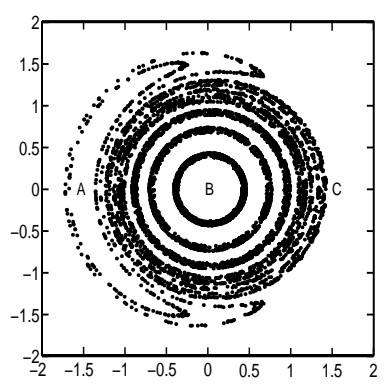

(a)

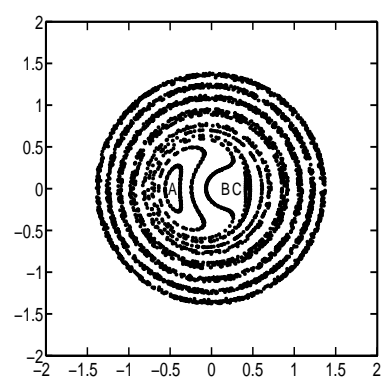

(b)
Figure 4: Phase space tracking of the RF phase modulation for (a) mayl8am, (b) mayl8pm. Here the horizontal and vertical axes mean $\sqrt{2 \tilde{J}} \cos \left(\tilde{\psi}-\nu_{m} \theta\right)$ and $\sqrt{2 \tilde{J}} \sin (\tilde{\psi}-$ $\left.\nu_{m} \theta\right)$, respectively.

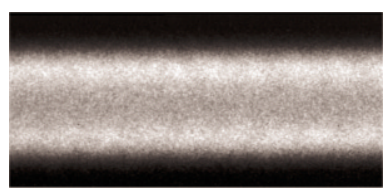

(a)

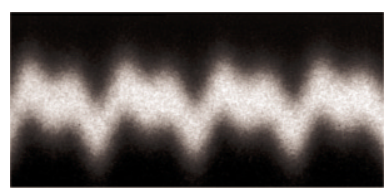

(b)
Figure 5: Streak camera images (a) when there is no RF noise-sideband. (b) when 10 hour beam lifetime reduction is generated due to RF noise-sidebands. Maximum horizontal time scale is $1 \mathrm{~ms}$, and vertical time scale which means the bunch length is same for two cases.

fore, the Touscheck lifetime which is directly proportional to the bunch length and gives the most limitation in the beam lifetime of the synchrotron light source facilities will be reduced at the dipole mode parametric resonance [2].

After considering small differences in the modulation frequency and the bifurcation frequency of mayl $18 \mathrm{pm}$, we can consider it as near the dipole mode parametric resonance status. Therefore, the strong longitudinal stability, the large reductions in the momentum spread, the bunch length or the beam lifetime can be obtained for may18pm as shown in Figs. 2, 3, and 4 [2]-[4]. This bunch length reduction is also well agreed with the streak camera images as shown in Fig. 5. Note that the noise frequency becomes far from the bifurcation frequency at the low beam current of may18pm of Fig. 3, the beam lifetime reduction is slowed down at the low beam current region.

Since the modulation frequency is well below the bifurcation frequency for mayl8am, the phase space area or the momentum spread is large as shown in Fig. 4(a). And its longitudinal damping and the beam lifetime reduction are small due to the weak resonance strength as shown in Figs. 2(b) and 3. In case of mayl $8 \mathrm{am}$, beams are reinjected at near $140 \mathrm{~mA}$ by the user's request.

Since there is only one stable fixed point at $f_{m}>f_{c}$, there is no net dipole mode phase oscillation in the bunches [2], [3]. As we increase the modulation frequency, the mo- mentum spread or the bunch length will be increased further. Therefore, we can obtain the longer Touscheck lifetime as well as the beam stability by adding a modulation signal with $f_{m} \simeq 2 f_{s}$ in the main RF signal [2]. But due to the bandwidth of the PLS RF phase loop of about $11 \mathrm{kHz}$, amplitudes of all RF noise-sidebands with higher than $11 \mathrm{kHz}$ will be substantially reduced when they are transfered to the RF cavity. Therefore, such a large increase in the Touscheck lifetime can not be generated by the RF noise driven phase modulation in the PLS storage ring. Since there is only one stable fixed point A for jun06am, the LCBMI is damped to the noise level, $0.003 \mathrm{deg} @ \mathrm{RF}$ as shown in Fig. 2(b). Although the momentum spread can be increased under the $f_{m}>f_{c}$ condition, according to simulation, there is little difference in the phase space area between mayl8pm and jun06am due to the smaller noise amplitude and $f_{m} \ll 2 f_{s}$ of jun06am [3]. Therefore, its beam lifetime is almost the same as that of mayl $18 \mathrm{pm}$ as shown in Fig. 3. However, one TCBMI is momentary generated by the strong longitudinally damped beams at much lower beam current than normal, $220 \mathrm{~mA}$ as shown in Fig. 2(b). This TCBMI with the mode number of 161 is due to the transverse $\mathrm{TM}_{110} \mathrm{~V}$ HOM $(826.4 \mathrm{MHz})$ of the PLS RF cavities and recorded by the LFS via the synchro-betatron coupling [1]. Although the TCBMI is generally weak, beams would be occasionally lost due to the transverse instability as shown in jun06am of Fig. 3.

\section{SUMMARY}

From measured results and simulation ones, we have found that the RF noise-sidebands of the low level RF system generate the dipole mode parametric resonance when one of them is near the bifurcation frequency. Due to the dipole mode parametric resonance, we have occasionally met the large reduction in the beam lifetime, the strong longitudinal damping, and the lowered threshold of the beam transverse instability. After reducing the RF noise by attaching additional mechanical phase shifter in the phase loop of the PLS RF system in 2000, we cured the abnormal phenomena.

\section{REFERENCES}

[1] Yujong Kim et al., submitted to IEEE Trans. Nucl. Sci., (September 2000).

[2] J. M. Byrd et al., Phys. Rev. E 57, 4706 (1998), S. Sakanaka et al., Phys. Rev. ST. Accel. Beams 3, 050701 (2000).

[3] Yujong Kim et al., "Bunch Length Beating in PLS Storage Ring," these Proceedings.

[4] A. Wu Chao and M. Tigner, Handbook of Accelerator Physics and Engineering, (World Scientific, Singapore, 1998), p. 187. 